\title{
STEPS TOWARDS MANUFACTURING DIGITISATION IN AN ATMOSPHERE FOR SMES
}

\section{BIBHUTI B PRADHAN}

Department of Management, Siksha 'O' Anusandhan (Deemed to be University), Bhubaneswar, Odisha, India

\begin{abstract}
This paper describes an EU-funded project, organized by a national Digital Innovation Hub (DIH) aimed at integrating the digitization of manufacturing operations in a growing SME. The improvements made by an Irish Precision Engineering SME towards incorporation of operator reporting are discussed, from manual to electronic format. The benefits of small changes are represented to the company via cost-effective methods. The technologies employed included components from the BEinCPPS architecture for MTConnect, IoT apps and open source applications.This enabled the acquisition of production data and the energy monitoring of machines from the factory floor to a central repository where data analysis tools developed key performance indicators (KPI's) for shop floor visualization and mobile dashboards. The experiment demonstrated how the use of commercial off-shelf technology, combined with the Enterprise Resource Planning (ERP) program of the plant, minimized routine production management activities, enhanced equipment and resources management while reducing the pressure on machine operators reporting.

KEYWORDS: Digitisation, Industry 4.0, BEinCPPS, FIWARE, MTConnect, Visualisation
\end{abstract}

Received: Jun 08, 2020; Accepted: Jun 28, 2020; Published: Aug 26, 2020; Paper Id.: IJMPERDJUN2020897

\section{INTRODUCTION}

The manufacturing sector in Europe employed 29.9 million people in 2015 with enterprises employing fewer than 250 persons, the Small to Medium Enterprises (SME's), representing $58 \%$ of total employment and $42 \%$ of total value added in 2016. Advancing technologies are driving what is being described as the 'fourth industrial revolution', which can bring with it the significant potential of transforming industries and deliver vital social economic and environmental impact. The greatest digital opportunity for Europe, is projected to be gained by the transformation of existing industry and enterprises. The term 'digitalisation' covers a wide range of possibilities regarding the extent of implementation of digital technologies. This can range from a basic level (such as the use of computers or the internet) and the use of modern technology (e.g. automation, cloud computing or big data) to progressive applications, such as adopting a business model based on digital products and services or using elements from Industry 4.0. It is expected that disruptive business models and enhanced production processes will generate new international market opportunities. Companies who have made the change perform better than their peers, studies indicate that productivity of the manufacturing sectors in Denmark, Finland and Sweden can be increased by approximately 15 percent, if automation in each branch is increased to the level of most automated countries[1], [2].

However smaller businesses are slow to change, with $41 \%$ of SME's in Europe not yet adopting the newer digital technologies and a third of German SME's still in the early stages of digitization. Despite the 
enormous economic potential of Industry 4.0, barriers exist to the widespread adoption of digitization and Industry 4.0 by SME's. SMEs, regardless of company size are still cautious about Industry 4.0 with relatively little use being made of large data streams to optimize processes and services. Due to lack of resources, SME's find it difficult to assess the technological maturity of relevant cloud and analytical solutions and their application to business. Management still lacks a methodical approach to business digitsation and a lack of standards and norms with regard to system and technology integration further delays investments in the integration of IT systems.

The drive by the European Commission to digitize European industry aims to help companies, researchers and public authorities make the most of new technologies. The measures taken, aim to link national and regional initiatives and of boosting investment through strategic partnerships and networks. Digital industrial platforms are crucial for digital transformation, as they bridge the gap between technology building blocks and industrial applications. Reference Architectures are high level abstraction definitions which help in the identification of challenges and concerns arising in various application scenarios[3]-[6], Several reference architectures have been proposed in the context of IoT and IIoT. Multi-layered approaches are favored. For connected Factories of the Future several initiatives have already started across the EU and beyond. Examples are RAMI, Virtual Fort Knox, FI-Ware based work inFITMAN and BEINCPPS, Industrial Data Space, IIRA, etc.

BEinCPPS (Business Experiments in Cyber Physical Production Systems) is funded under the European Commission's Horizon 2020 Framework Program for Research and Innovation through the Factories of the Future Call for Proposals (H2020-FoF-2015). It addresses the Topic "ICT Innovation for Manufacturing SMEs (I4MS)" to expand the digital innovation of manufacturing SMEs in Europe. The BEinCPPS 3-layer architecture of (machine, factory, cloud) was developed from state-of-the-art advances in the fields of Internet of Things, Future Internet and CPS / Smart Systems. It is capable of supporting the development of innovative applications via open APIs to integrate existing legacy hardware and software systems via open standards.

\section{PROJECT IMPLEMENTATION}

\section{Project Problem Context}

The manufacturing site for this case study is a precision engineering SME involved in the sub-contracted precision engineering manufacture for organisations in the Medical Device and Aeronautical industries. At the start of the project an analysis of the production value streams was carried out to identify where the greatest benefit would resultthrough digitization within the production process. The areas identified were as follows: Part Program Version Control, Job Scheduling and Local/Remote Visualization of machine and Work Order Status.

The SME operates a range of un-networked CNC machines and lathes of differing manufacturer types and capacities. Parts are designed by the in-house design team, then scheduled for production by operators on the factory floor by the production management team. The Manufacturing Company implements ISO 9001, ISO 14001, ISO 13485 and where applicable FDA regulations as well as multiple process specifications for the Aerospace Industry[7]-[9]. The implementation of these processes on the factory floor are paper based and driven by Microsoft Excel charts.

Operators have, in the past, re-used CNC part codes stored on the machines, which have been used for previous production runs. This, however, may mean new design changes are not included. The SME also has the ability to deliver a fast turnaround for valued customers, which is positive for revenue, but can negatively impact scheduling as work orders 
may get interrupted as new high priority orders come in. When work orders are completed, operators enter the production details into the company enterprise resource planning (ERP) system through a shared PC. The data entry process is cumbersome; as many screens need to be accessed on to complete the process, resulting in untimely and often inaccurate data. The production manager's ability to accurately perform job scheduling tasks was hampered by the following problems.

- The generation and maintenance of the weekly production schedule requires greater than 2.5 person days.

- Production Schedule management is delivery date driven.

- Customer re-prioritisation of work orders occurs on a regular basis requiring updates to the schedule.

- Operator reporting on production activity is often late and inaccurate.

\section{PROBLEM GOAL}

The goal of the project was to develop a Cyber Physical System (CPS), which integrates with the enterprise ERP system to provide production stakeholders with up to date production data generated on the factory floor. The key problem areas addressed by this project are Manufacturing Logistics and Productivity. The work was carried out in a partnership between the Digital Hub and an automation integration company. The aim of the project was to deliver a digitization solution which would start the SME on the way towards making better use of available data within the organization[10]-[12]. Components from the BEinCPPS platform were used to streamline the flow of data from factory floor to cloud. The components were chosen for their flexibility and ease of integration with data sources within the factory and with a view towards future expansion of data sources, analysis and possible visualization requirements.

The MD4PROD (Manufacturing Digitization for Production and Resource Optimisation) experiment designed and implemented the required CPS which facilitated the digitization and use of production and operational resource data within a workcell operating 4 Doosan CNC machines. The 4 CNC machines were connected to the Factory Network. Industrial grade power-over-Ethernet Touch Screen Tablets and bar-code scanners were installed at each CNC machine. A smart TV screen was placed in the workcell area to provide direct feedback to the operators and to display each work order status. The project uses the three-layers of the BEinCPPS architecture (cloud, factory, field or machine). Data is captured at machine level for integration with the ERP (M1) system to generate relevant KPI values.

\section{Project Architecture}

The system architecture used is shown below in Figure 1. The Factory Informer application was the core process used in the capture of machine status as well as user related data input. As the project required the acquisition of data from specific varieties of CNC machines, which are in wide use across the Precision Engineering Industry an open source standard toolset, was used to allow data access in a non-proprietary format. MTConnect was chosen as it provided the access required with the Manufacturer machines in question.

Data management was controlled using the FIWARE Orion Context Broker acting as a broker for data notifications between producers and subscribers. Orion is a C++ implementation of the NGSIv2 REST API binding developed as a part of the FIWARE platform. Using the broker, the complete lifecycle of context information (update, query, registration and subscription) can be managed. By subscribing to context information, notification is received when context elements have changed simplifying the communications and management required of IoT related systems. The 
Factory Informer process was designed to transmit directly to the broker using the NGSIv2 API. This simplified the software requirements and allowed focus on developing the ability to communicate with more CNC machine types using direct API calls as opposed to requiring OMP-UA adapters for each machine type.

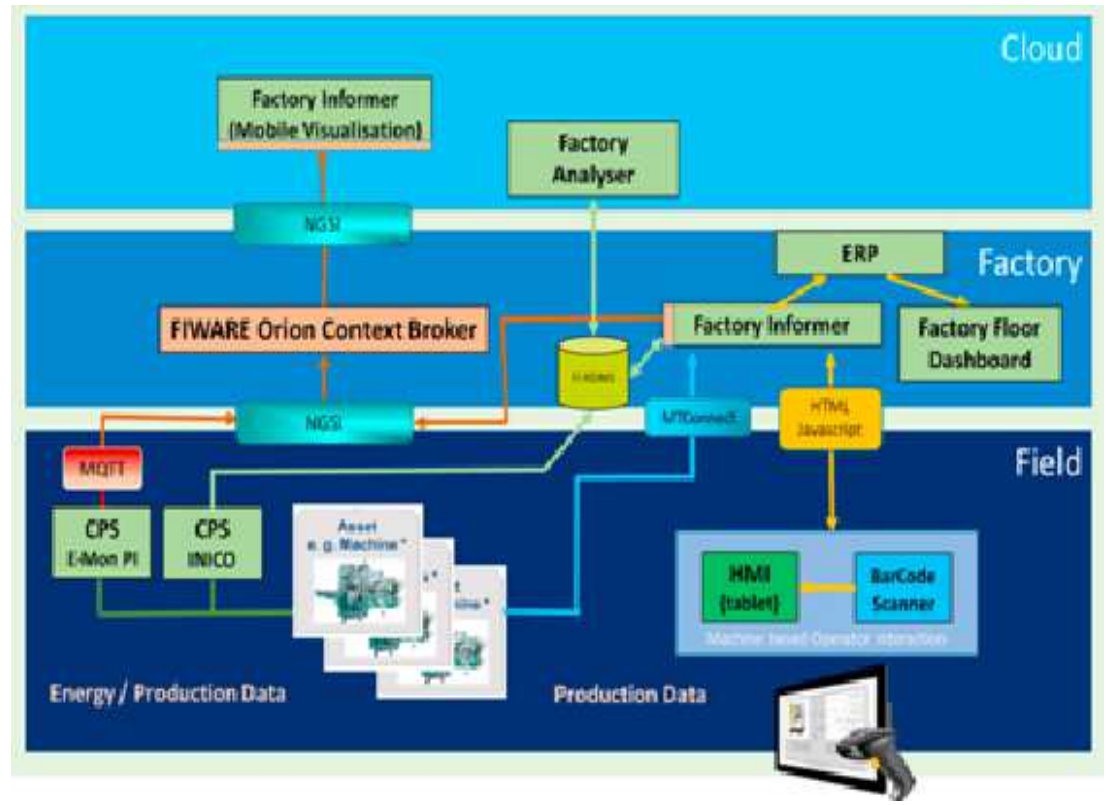

Figure 1: System Architecture.

The logging of energy use by legacy machines is performed using an Inico Tech based PLC CPS. The data flow from the energy monitoring CPS transmits directly to the FI-RDBMS installation. The Microsoft.NET Framework3.5 using Microsoft Visual Studio Development tools and Microsoft SQL Server was used to be compatible with the M1 ERP system and existing IT infrastructure.

The BEinCPPS components used in the MD4PROD experiment as shown in Figure 1 above, enable the transmission of data from the device layer to the Orion Context Broker through to the visualization layer. Using the CPPS Publishing Services component it was possible to allow the transfer of data in a relatively simple manner from the machine level to the Orion Context Broker. The Factory Informer process was enhanced by programming it to send NGSI messages to the Orion Context Broker, this facilitated the availability of machine and production job status on a near real time basis for visualization to relevant personnel. Using the IoT UL 2.0 Agent, changes in machine status, captured using a proximity sensor connected to a Raspberry Pi were transferred to the Orion Context Broker.

Visualization of the status of each machine and the relevant job status was provided in various formats throughout the site for Production Management and operators using Freeboard. This information was also available on a mobile application allowing management have remote access to data when required.

\section{Operator Engagement}

Using the newly installed system, each operator logs into the ERP system using a simplified one-step interface on the touch screen tablet. The work-order job card is scanned using the barcode scanner. This signals the start of a work order to the Factory Informer process as illustrated below on Figure 2. The download of the correct set of CNC machine part program codes from a version control repository is then triggered preventing the use of out of date partcode files and ensures data 
integrity. The Factory Informer process using the MTConnect API detects the start of the work batch, on the CNC machine and updates the work order job status on the M1 ERP system.

When the work order is completed, the operator enters the number of parts successfully completed as well as the number if any of scrap parts. This updates the ERP system to report the completion of the job. The status of each work order for the workcell is displayed on a smart TV screen.

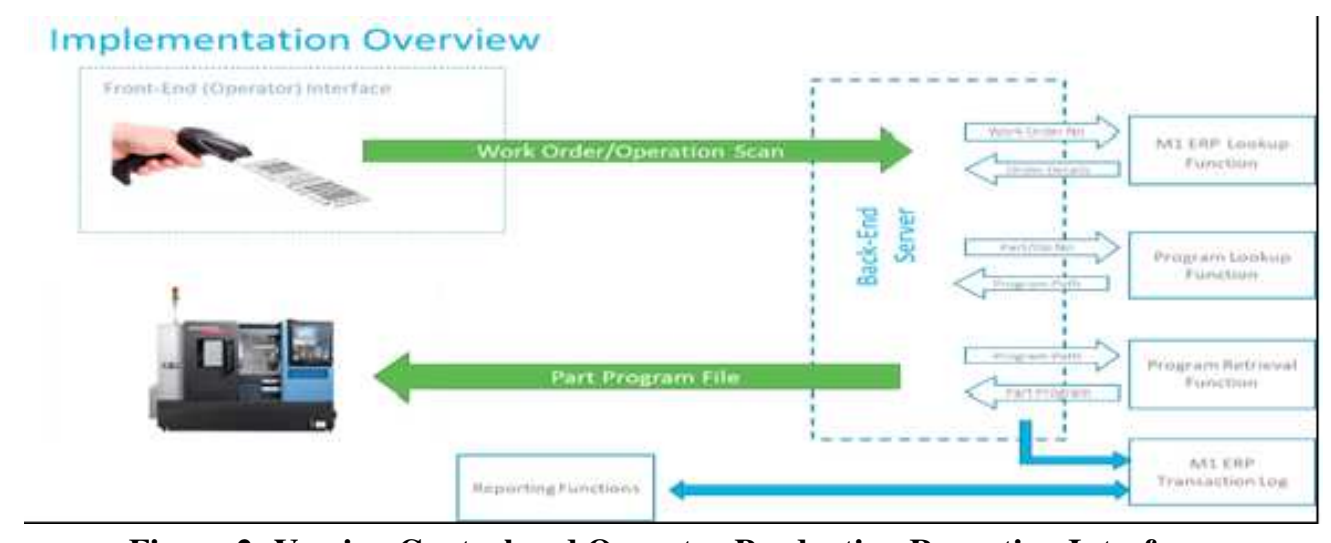

Figure 2: Version Control and Operator Production Reporting Interface.

The workcell TV screen is updated to indicate the upcoming jobs in the workcell, and jobs are removed from the screen as they are completed. Management has reported that the removal of jobs from the list of active jobs provides operators with motivation to enter data in a timely manner.

\section{Remote Machine State Visualization}

Changes in machine state are detected using the MTConnect API by the Factory Informer process. By updating the Orion Context Broker of state changes, any visualization tools or remote display applications are automatically notified. Information is displayed using a smart TV in the Design team office is available on a mobile phone application for remote monitoring. The duration of a state change is clearly shown which incentivizes operators and management to get machines operating as soon as possible.

Engineering staff have a clear view of the status of each machine in the workcell using a TV mounted on a wall in their office. This alerts management to ensure that Management and Maintenance personnel react in an appropriate manner. Using the Factory Informer Mobile App, an up-to date view of the status of each machine in the workcell is available to Production Management. Scrolling through the list of machines displayed, the status of each machine is immediately visible. A color-coded background indicates the most important condition for the Production Manager .e. the status of the machine itself. The length of time the machine is in this mode is also displayed. This allows the manager to react in an appropriate way in each case.

\section{Legacy Machine Monitoring}

Through a needs assessment of the Production SME it was identified that data-driven strategies based on real-time and historical information could hold great potential to help the Company to better understand their business and to optimise performance. One source of data is held in the energy signatures of productive equipment, which was logged using the Inico Tech based cyber-physical system (CPS) energy monitoring system. The FactoryAnalyser App was developed to 
demonstrate this potential. Energy signature data or 'Power Profiles' can be captured by a CPS as Time Series Datasets (TSDs), stored as values with chronological time-stamps, analysed through algorithms and the data presented on tablets or on the Cloud to inform the decision making of the Company. It is also possible through the analysis of energy signature to generate very accurate production information, particularly for legacy machines, where integrated production reporting may not be available. This adds significant value to the development of a CPS for machine energy on the factory floor and provides an empirical view on real and verifiable production activity.

\section{RESULTS}

The digitization project delivered improved Operator reporting engagement through easier to enter production data (less logging steps involved) with on-time and more accurate reporting of production metrics. Through precise knowledge of the number of quality/scrapped parts produced and the automated capture of machine status and operational parameters by networking of machines, gaps in reporting have been eliminated for the workcell with the 4 CNC machines on which the project was implemented.

This has facilitated improved Production Scheduling and Overall Equipment Effectiveness (OEE) due to up to date knowledge of machine and production status. Near real-time production data is now available on the factory ERP system for use by Production Management in managing daily production operations, which is of significant value to the Company Management.

The identification of individual parts through analysis of energy profile data for legacy machines on another workcell deliver an automated method of classifying cycle times, machine utilisation and the added-value component of the energy consumption of legacy machines.

The values shown below in Table 1 are from an older CNC machine not having an intelligent controller. Previously this data was manually reported by operators and often included delays and errors. The availability of accurate data such as the number of parts made and the percentage of time the machine has been productive inform on stock levels and enable management teams to put greater focus on improving OEE on these older machines. Having actual data on the actual percentage of productive time $(15.8 \%)$ brings focus on the need to improve the operations on these machines.

Table 1: Production and Process Indicators of Legacy Machines

\begin{tabular}{|l|c|}
\hline \multicolumn{1}{|c|}{ Indicator } & Total Values \\
\hline Parts Made & 2,217 \\
\hline Real Cycle Time (Seconds) & 374.7 \\
\hline Total Energy Consumption (kWh) & $5,051.98$ \\
\hline Consumption (kWh) & 2,580 \\
\hline Non-Productive Energy Consumption (kWh) & $2,471.14(48.9 \%)$ \\
\hline Total Working Time (Hours) & $1,411.79$ \\
\hline Total Working Time (Hours) & $1,411.79$ \\
\hline
\end{tabular}

Easier and thus better Operator reporting of work order status, with production and scrap quantities have resulted in: improved delivery dates and better customer satisfaction. Improved 'On time Delivery' a metric calculated by their main customer has increased from less than $80 \%$ to greater than $90 \%$ since operators have started using the new system. 


\section{CONCLUSIONS}

The MD4PROD project, introduced a Cyber Physical Framework that enables the digitization and use of production and organizational resource data in a manufacturing environment for precision engineering. The project has allowed changes in the recording of production operation, allowing the Production Management team to switch from a paper-based reporting method, which has often been slow and unreliable, to near-real-time monitoring of work order progress and the quantities of product parts produced on a value stream. Management is invaluable in the ability to show the near-real-time status of machinery and work orders, because it makes greater overall utilization of equipment and facilitates better production schedule.

Potential updates to incorporate predictive or reactive maintenance functions should provide more in-depth system operating statistics such as spindle speeds and running hours. The incorporation of the feature on other production process areas such as Products Inwards, Inspection and Dispatch is expected. This will give customers access to an up-to-date report on the status of work orders and the amount of stock available. Reports of this type are currently performed manually for several clients each week and for some clients on a regular basis. The opportunity to deliver such data directly to clients would free employees from reporting duties. The system will have maximum 360 controls over the entire manufacturing process when complete.

\section{REFERENCES}

1. B. Merrilees, S. Rundle-Thiele, and A. Lye, "Marketing capabilities: Antecedents and implications for B2B SME performance," Ind. Mark. Manag., 2011.

2. European Commision, "User guide to the SME Definition," 2015.

3. A. Gilmore, "Entrepreneurial and SME marketing," Journal of Research in Marketing and Entrepreneurship. 2011.

4. L. Lloyd-Reason, K. Ibeh, and B. Deprey, “Top Barriers and Drivers to SME Internationalisation,” 2009.

5. N. A. Rahman, Z. Yaacob, and R. M. Radzi, “The Challenges Among Malaysian SME: A Theoretical Perspective," World J. Soc. Sci., 2016.

6. Q. Zhao, H. J. Qi, and T. Xie, "Recent progress in shape memory polymer: New behavior, enabling materials, and mechanistic understanding," Progress in Polymer Science. 2015.

7. T. von Leipzig et al., "Initialising Customer-orientated Digital Transformation in Enterprises,” Procedia Manuf., 2017.

8. T. D. Oesterreich and F. Teuteberg, "Understanding the implications of digitisation and automation in the context of Industry 4.0: A triangulation approach and elements of a research agenda for the construction industry," Computers in Industry. 2016.

9. A. Kusiak, “Smart manufacturing,” Int. J. Prod. Res., 2018.

10. H. Kroll, D. Horvat, and A. Jäger, “Effects of Automatisation and Digitalisation on Manufacturing Companies' Production Efficiency and Innovation Performance,” Fraunhofer ISI Discuss. Pap. Innov. Syst. Policy Anal., 2018.

11. V. A. Prabhu, M. Elkington, D. Crowley, A. Tiwari, and C. Ward, "Digitisation of manual composite layup task knowledge using gaming technology," Compos. Part B Eng., 2017.

12. Plattform Industrie 4.0, “What is Industrie 4.0?,” Federal Ministry for Economic Affairs and Energy, 2017.. 
